\title{
Lexical Influences on the Perception of Sarcasm
}

\author{
Roger J. Kreuz \\ Department of Psychology \\ The University of Memphis \\ Memphis, TN 38152 \\ rkreuz@memph is . edu
}

\author{
Gina M. Caucci \\ Department of Psychology \\ The University of Memphis \\ Memphis, TN 38152 \\ gcaucci@memphis.edu
}

\begin{abstract}
Speakers and listeners make use of a variety of pragmatic factors to produce and identify sarcastic statements. It is also possible that lexical factors play a role, although this possibility has not been investigated previously. College students were asked to read excerpts from published works that originally contained the phrase said sarcastically, although the word sarcastically was deleted. The participants rated the characters' statements in these excerpts as more likely to be sarcastic than those from similar excerpts that did not originally contain the word sarcastically. The use of interjections, such as gee or gosh, predicted a significant amount of the variance in the participants' ratings of sarcastic intent. This outcome suggests that sarcastic statements may be more formulaic than previously realized. It also suggests that computer software could be written to recognize such lexical factors, greatly increasing the likelihood that nonliteral intent could be correctly interpreted by such programs, even if they are unable to identify the pragmatic components of nonliteral language.
\end{abstract}

\section{Introduction}

It has long been assumed that verbal irony, or sarcasm, is principally a pragmatic phenomenon, and many studies from psycholinguistics have demon- strated the social, contextual, and interpersonal factors that affect its use and interpretation (for reviews, see Gibbs, 1994, 2003; Giora, 2003).

An example of such a pragmatic factor is common ground (Clark, 1996). The more familiar two people are with one other, the more likely it is that they will employ sarcasm (Kreuz, 1996). When interlocutors in a story share common ground, experimental participants read sarcastic statements more quickly, and are more certain of the sarcastic intent, than when the interlocutors share little common ground (Kreuz and Link, 2002). These results can be explained in terms of a principle of inferability: speakers will only employ sarcasm if they are reasonably certain that their hearers will interpret it correctly (Kreuz, 1996).

Such results have led to pessimistic forecasts concerning the likelihood that computer programs would ever be able to understand nonliteral language (e.g., Dews and Winner, 1997). If the use of such language relies solely on pragmatic factors, it would indeed be a considerable challenge to create software that could detect and interpret it.

One difficulty with this conclusion is that most psycholinguistic studies of sarcasm have used experimenter-generated materials instead of actual utterances. For example, sarcastic statements are often hyperbolic (Kreuz and Roberts, 1995), and so researchers have typically employed extreme constructions, such as What perfectly lovely weather! as sarcastic commentary on a sudden downpour (Kreuz and Glucksberg, 1989).

Such research, however, has unintentionally confounded the pragmatic and the lexical aspects of sarcasm. It may be the case that particular words or collocations (e.g., perfectly lovely) serve as a cue 
for sarcasm by themselves. Previous research has not attempted to tease apart these lexical and pragmatic factors, even though the importance of lexical factors has been suggested previously. Kreuz and Roberts (1995) proposed that collocations consisting of extreme adjectives and adverbs (e.g., simply amazing, or absolutely fantastic) may serve as a conventional way of signaling ironic intent. This idea has been expanded by Utsumi (2000), who suggested that such verbal cues provide a way of implicitly displaying negative attitudes via sarcasm.

Of course, interlocutors in face-to-face conversations can rely upon both verbal and nonverbal cues to signal ironic intent (e.g., rolling of the eyes, heavy stress, or slow speaking rate). The authors of narratives must cue their readers without recourse to such conventions. The methods used by authors, therefore, might provide a way to assess the contribution of lexical factors to the perception of sarcasm.

The goal of the present research was to determine whether specific lexical factors (e.g., the use of certain parts of speech, or punctuation) reliably predict readers' perceptions of sarcasm. Unlike most previous research on sarcasm, the experimental materials were drawn from published narratives.

\section{Method}

Participants were asked to read excerpts from longer narratives, and then to rate how likely it was that the speaker was being sarcastic.

\subsection{Materials}

Google Book Search was used to find instances of the phrase said sarcastically. This resource, formerly known as Google Print, contains more than 100,000 published works that are either in the public domain, or have been provided by publishers. A wide variety of genres is represented (e.g., historical novels, romance novels, and science fiction).

The phrase said sarcastically was found hundreds of times in the corpus, and 100 of these instances were randomly selected for the study. Fifteen control texts were also selected at random from the Google Book Search corpus. Five of the control items contained the phrase I said, five contained he said, and five contained she said.
In order to create experimental materials, we excerpted the entire paragraph that the key phrase appeared in, as well as the two paragraphs of context appearing above and below. The excerpts varied considerably in length, but the mean length for the 115 excerpts was 110 words $(S D=58)$.

The phrase that the collocation said sarcastically referred to was emphasized in bold-faced type. If the phrase appeared at the end of a sentence, only the words that occurred before it within quotation marks were made bold. If the sentence continued after the phrase said sarcastically, the following words in quotation marks were also made bold. Finally, the word sarcastically was removed, leaving just the phrase [speaker] said. The speakers' statements in the control excerpts were made bold using the same procedure, ensuring that the two sets of excerpts were identical in appearance. The mean length of the bold-faced phrases for all the excerpts was 6.45 words $(S D=8.05)$.

Each excerpt was printed on a separate page, along with three questions. The first question asked How likely is it that the speaker was being sarcastic? A seven-point scale, with endpoints labeled not at all likely and very likely, appeared below the question. A second question asked Why do you think so? Two blank lines were provided for the participants' responses. Finally, the participants were asked How certain are you that the speaker was being sarcastic? A seven-point scale, with endpoints labeled not at all certain and very certain, appeared below the question.

Five different sets of sarcasm excerpts (20 per set) were created. Booklets were constructed by randomly interspersing the subset of sarcasm excerpts with all of the control excerpts. The order of pages was randomized for each participant.

\subsection{Coding}

Two judges independently coded the excerpts on three dimensions:

(1) Presence of adjectives and adverbs. Following Kreuz and Roberts (1995) and Utsumi (2000), the judges identified the use of adjectives or adverbs in the bold-faced segments of each excerpt. The coding was binary: 0 for none, and 1 for one or more adjectives and adverbs.

(2) Presence of interjections. Certain terms, such as gee or gosh, are used for the expression of emotion, and may also serve as a cue for nonliteral 
intent. The excerpts were again coded in a binary fashion.

(3) Use of punctuation. Exclamation points indicate emphasis, which may be a signal of nonliteral intent. Question marks are used in tag questions (e.g., You really showed him, didn't you?), which are often rhetorical and nonliteral (Kreuz et al., 1999). The use of either an exclamation point or question mark was coded in a binary fashion.

The agreement between the judges' coding was $95 \%$ across all excerpts. The small number of disagreements was primarily the result of variability in how dictionaries define interjections. All disagreements were resolved through discussion.

\subsection{Procedure}

Participants were 101 undergraduates at a large public university. They received course credit for their participation. The participants were tested in small groups, and asked to work through the booklets at their own pace. Each participant read and answered questions for 35 excerpts: 20 sarcasm excerpts, and all 15 control excerpts (only a subset of the sarcasm materials was given to each participant to offset fatigue effects).

The term sarcasm was not defined for the participants, and they were asked to rely solely on their intuitive understanding of the term. (Previous research with the same population suggests that a fairly high level of agreement exists for the concept of sarcasm; see Kreuz, Dress, and Link, 2006).

\section{Results}

Only the responses from the first question (likelihood that the speaker is being sarcastic) will be discussed here. For each participant, a mean score for the 100 sarcasm and 15 control excerpts was computed. As expected, the sarcasm excerpts received higher scores $(M=4.85, S D=.67)$ than the control excerpts $(M=2.89, S D=.86)$, and the difference was significant, $t(100)=19.35, p<.001$. This means that the participants had sufficient context for determining sarcastic intent in the test excerpts, and that the participants were able to distinguish between the two groups of excerpts.

To determine the relative importance of the lexical factors on the perception of sarcasm, a regres- sion analysis was performed. The criterion variable was the mean sarcasm rating for each excerpt. Five predictor variables were employed: (1) the number of words in each excerpt, (2) the number of boldfaced words in each excerpt, (3) the presence of adjectives and adverbs, (4) the presence of interjections, and (5) the use of exclamation points and question marks. Variables 3 to 5 were coded in a binary fashion, as described in section 2.2. Ratings for both the sarcastic and the control excerpts were entered.

The number of words and number of bold-faced words are theoretically uninteresting variables, so they were forced into the equation first as one block. The three predictor variables of interest were entered in a second block using a stepwise method.

The first block, containing the two length variables, failed to account for a significant amount of the variance, $F(2,112)=1.37$, n.s., $R^{2}=.024$. This was a desirable outcome, because it meant that participants were not influenced in their judgments by the lengths of the excerpts they were reading, with longer excerpts providing more contextual cues.

For the second block with the three variables of interest, only the presence of interjections entered into the equation, $F(1,111)=6.10, p=.015, R^{2}=$ .051 . The presence of adjectives and adverbs, and the use of punctuation, failed to predict a significant amount of the variance in the participants' ratings of sarcastic intent.

\section{Discussion}

Previous theory and research has largely ignored the potential role of lexical factors in the delivery and detection of sarcasm. This bias has been reinforced by the use of experimenter-generated materials that may have obscured the contributions made by these factors. This study is the first to assess the importance of such lexical factors, using ecologically valid materials.

On the one hand, the amount of variance accounted for by lexical factors was rather small: just $5 \%$. On the other hand, it must be remembered that the excerpts themselves were taken from booklength works, so the participants only had a fraction of the original context with which to determine the intent of the (potentially) sarcastic statement. Nevertheless, the participants were able to reliably differentiate between the sarcastic and 
control excerpts, which suggests that specific local factors were influencing their judgments.

In addition, it must be remembered that only a small number of lexical factors was assessed, and in a fairly coarse way (i.e., with binary coding). Out of just three such factors, however, the use of interjections was a significant predictor of the participants' ratings. An inspection of the excerpts suggests that certain formulaic expressions (e.g., thanks a lot, good job), foreign terms (e.g., au contraire), rhetorical statements (e.g., tell us what you really think), and repetitions (e.g., perfect, just perfect) are also common in sarcastic statements. However, the set of excerpts was not large enough to allow an analysis of these expressions. A large online corpus would permit the identification of many such collocations, but determining whether the phrases were actually intended sarcastically would be more difficult than in the present study.

One could argue that the use of the phrase said sarcastically reflects poorly on the authors themselves. Ideally, a writer would not need to be so explicit about a character's intentions: it should be clear from the context that the statement was intended nonliterally. However, an author is writing for an indeterminate audience that may exist in the present or in some future time. It should not be surprising, therefore, that authors occasionally feel the need to use such a phrase, and this reflects how difficult it is to communicate nonliteral intent clearly.

It should also be noted, however, that some of the authors used the word sarcastically rather broadly, as a synonym for angrily or jokingly, even when the statement was intended literally. This suggests that the use of this term may be undergoing some change (see Nunberg, 2001 for a similar claim).

Finally, these results have important implications for software programs that attempt to "understand" natural language. Nonliteral language presents formidable challenges for such programs, since a one-to-one mapping of words to meaning will not lead to a correct interpretation (e.g., Gee, I just love spending time waiting in line). However, the present results suggest that, in some contexts, the use of interjections, and perhaps other textual factors, may provide reliable cues for identifying sarcastic intent.

\section{References}

Herbert H. Clark, 1996. Using Language. Cambridge: Cambridge University Press.

Shelly Dews and Ellen Winner, 1997. Attributing meaning to deliberately false utterances: The case of irony. In C. Mandell and A. McCabe (Eds.), The Problem of Meaning: Behavioral and Cognitive Perspectives. Amsterdam: Elsevier.

Raymond W. Gibbs, Jr., 1994. The Poetics of Mind: Figurative Thought, Language, and Understanding. Cambridge: Cambridge University Press.

Raymond W. Gibbs, Jr., 2003. Nonliteral speech acts in text and discourse. In A. C. Graesser, M. A. Gernsbacher, and S. R. Goldman (Eds.), Handbook of Discourse Processes (pp. 357-393). Mahwah, NJ: Lawrence Erlbaum Associates.

Rachel Giora, 2003. On our Mind: Salience, Context, and Figurative Language. New York: Oxford University Press.

Roger J. Kreuz, 1996. The use of verbal irony: Cues and constraints. In J. S. Mio and A. N. Katz (Eds.), Metaphor: Implications and Applications (pp. 23-38). Mahwah, NJ: Lawrence Erlbaum Associates

Roger J. Kreuz, Megan L. Dress, and Kristen E. Link, 2006, July. Regional Differences in the Spontaneous Use of Sarcasm. Paper presented at the annual meeting of the Society for Text and Discourse, Minneapolis, $\mathrm{MN}$.

Roger J. Kreuz and Sam Glucksberg, 1989. How to be sarcastic: The echoic reminder theory of verbal irony. Journal of Experimental Psychology: General, 118:374-386.

Roger J. Kreuz, Max A. Kassler, Lori Coppenrath, and Bonnie McLain Allen, 1999. Tag questions and common ground effects in the perception of verbal irony. Journal of Pragmatics, 31:1685-1700.

Roger J. Kreuz and Kristen E. Link, 2002. Asymmetries in the use of verbal irony. Journal of Language and Social Psychology, 21:127-143.

Roger J. Kreuz and Richard M. Roberts, 1995. Two cues for verbal irony: Hyperbole and the ironic tone of voice. Metaphor and Symbolic Activity, 10:21-31.

Geoffrey Nunberg, 2001. The Way we Talk Now: Commentaries on Language and Culture. Boston: Houghton Mifflin

Akira Utsumi, 2000. Verbal irony as implicit display of ironic environment: Distinguishing ironic utterances from nonirony. Journal of Pragmatics, 32:17771806. 